\title{
Risk assessment of Internet Finance under the background of big data
}

\author{
$\mathrm{Ju}$ Zhuoru \\ School of Mathematics, Southwest Jiaotong University, Chengdu, Sichuan, 610000 \\ 734380844@qq.com
}

\begin{abstract}
:
At present, it is an era of economic globalization. The development of China's Internet financial industry should keep pace with the times and keep pace with the times. Compared with the developed countries in the west, the development level of China's Internet financial industry is relatively backward, fully protecting the security of private capital funds. Therefore, Internet financial enterprises should actively respond to the call of the national policy and increase investment in financial risk control. The introduction and application of advanced big data technology, strengthen the judgment and analysis of financial risk information, and control the risk of enterprise investment management within a reasonable range.
\end{abstract}

Keywords: VaR; historical simulation method; K-means clustering

\section{Introduction}

Since the 80 s of last century, the rapid development and innovation of the global economy and finance has brought fierce competition to the global financial field. The risks are obvious [1]. As long as there are financial activities, there will be risks. Once the financial risks happen, it will lead to the failure of the entire financial system to operate properly, and the social and economic order will also be affected. Moreover, it may lead to serious political crises and wars.

Financial risks can be divided into many kinds. Generally speaking, interest rate risk, exchange rate risk and securities and futures risks are common. Financial risks will result in financial crisis, financial system disorder, financial institutions successive failures, interest rate fluctuations, exchange rate fall and capital flight [2]. It has even brought deeper economic crises. In recent years, these financial risks brought about a series of crises. The emergence of these crises made governments and financial institutions pay more and more attention to risks. China is a developing country, and the financial market is also a new financial market. The regulatory system for financial assets is not mature enough [3]. Therefore, there are some problems in China's financial market, such as low degree of market liberalization, imperfect market structure and strong market speculation. In order to prevent the spread of risks, the government has taken some corresponding measures to control the abnormal situation of the stock market [4].

\section{Estimation of VaR and conditional value at risk}

\subsection{Definition of VaR and CVaR}

Before the advent of $\mathrm{VaR}$, the mainstream risk measurement methods mainly rely on sensitivity and volatility methods. However, either way, there are problems and shortcomings that are difficult to solve. Therefore, in order to solve the problems that traditional risk measurement methods can not solve, a tool that can comprehensively measure the market risk of complex portfolio VaR is produced.

The VaR method is first proposed by JP Morgan. Its meaning is "the value at risk". It refers to the maximum possible loss of a financial asset or portfolio under the normal fluctuation of the market. The more accurate statement is that the maximum loss of a financial asset portfolio in a specific period of time under certain confidence level is expressed as follows:

$$
\operatorname{Pr} o b(\Delta p>\operatorname{VaR})=1-\alpha
$$

Among them, Prob indicates that the probability of asset value loss is less than the upper limit of possible loss. $\quad p$ for a certain financial asset in a certain holding period of value loss, VaR is the level of the fixed letter. $\alpha$ possible upper limit of loss. $\alpha$ is a given confidence 
level, for example, within a $99 \%$ confidence level of a financial institution, that is, $\alpha=0.99$ calculated $\mathrm{VaR}$ value equals 15 million, meaning that the probability that the mechanism loses more than 15 million in the corresponding time is less than or equal to $1 \%$, so that the mathematical expression of $\mathrm{VaR}$ can be obtained as follows:

$$
\begin{aligned}
& V_{\alpha} R_{\alpha}=\inf \{l \in R: P(L>l) \leq 1-\alpha\} \\
& =\inf \left\{l \in R: F_{L}(l) \geq \alpha\right\}
\end{aligned}
$$

$\mathrm{VaR}$ is also a certain confidence level a (for example $95 \%$ ), the biggest loss faced by the investment portfolio. Specifically, we define the 1-A percentile of the return distribution $V a R$ :

$$
\operatorname{VaR}(a)=-F_{r}^{-1}(1-a)
$$

among $F_{r}(\square)$ is the cumulative distribution function of portfolio returns.

The definition above is defined from the left tail of the income distribution, and it is often necessary to define VaR: from the right side of the distribution of earnings.

$$
\operatorname{VaR}(a)=F_{r}^{-1}(1-a)
$$

Because VaR does not have sub additivity, in other words, the VaR value of portfolio investment may exceed the weighted average $\mathrm{VaR}$ of assets in the portfolio. Therefore, in practical applications, CVaR with multiple additivity is often used to measure the risk of portfolio. CVaR measures the average loss when loss exceeds VaR at a certain confidence level a. The specific definition is as follows:

$$
C \operatorname{VaR}(a)=-E[r \mid r \leq-V a R]=\frac{\int_{-\infty}^{-V a R} z f_{r}(z) d z}{F_{r}(-V a R)}=\frac{\int_{-\infty}^{-V a R} z f_{r}(z) d z}{1-a}
$$

At the same time, you can define CVaR: From the angle of right tail.

$$
\operatorname{CVaR}(a)=\frac{\int_{V a R}^{\infty} z f_{r}(z) d z}{1-a}
$$

Among them: $f_{r}(\square)$ is the probability density function of portfolio returns.

To sum up, the VaR value calculated in the value at risk model is in essence a single critical value of a certain asset value lost in a holding period under a certain confidence level. In practice, it is embodied in the amount at which the financial institutions are trading at a critical point. From the above formula, we can see that the VaR value is related to two parameters, one is the holding period of assets $t$. And the other is the significance level of computation $\alpha$. The following are the important factors that affect these two parameters.

(1) Holding period choice

The holding period is to calculate the time range of VaR. Generally speaking, the holding period is one day or one month. The longer the holding period is, the greater the uncertainty in the future. As a result, the risk of securities held by securities holders will increase and volatility will increase, so the VaR value of a longer holding period will also increase correspondingly. The following factors are usually considered: liquidity, normality, position adjustment and data constraints.

(2) Confidence level selection

The choice of confidence level depends on the need for $\mathrm{VaR}$ validation, internal venture capital requirements, regulatory requirements and the need for comparison among different institutions.

\subsection{VaR and CVaR based on historical simulation}

The historical simulation method belongs to the full valuation method without assuming the distribution. The precondition is that the future risk situation and the past risk situation can be duplicated in the future. The future risk change can be simulated by collecting all the risk information in the past period.

(1) Choose the appropriate period of observation and confidence level.

(2) During the observation period, risk factors historical yield time series are arranged in ascending order.

(3) The data of the inspection period are put in the historical data, and the time series obtained in the first step are arranged.

VaR and CVaR based on historical simulation method are used to calculate VaR and CVaR. using the quantile of empirical distribution of historical value of portfolio return. This method can be used to calculate the change value of VaR and CVaR in the past 5 years in the balance Bao. It can be concluded that the risk of balance treasure is relatively low at the beginning, but as time goes on, the rate of return decreases rapidly, which is consistent with the actual situation. With the rapid growth of funds, it is difficult for the balance treasure to guarantee a very high yield. Therefore, the balance treasure is only suitable for small investment, and it is not recommended to put a large amount of money into the balance treasure. Its main advantage is good flexibility, but low yield.

\subsection{VaR and CVaR based on normal distribution}

Many financial models are based on the assumption that returns are subject to normal distribution. In practice, normal distribution is often used as a reference benchmark for analysis. If returns are subject to normal distribution, we will carry out corresponding VaR and CVaR calculations. We still choose 10000 profit data of $\mathrm{Yu}$ Bao Bao in the past 5 years, and draw a general trend 
similar to that of historical simulation method. At the same time, the value of $\mathrm{VaR}$ is slightly larger than $\mathrm{CVaR}$, which is also in line with the actual situation.

\subsection{VaR and CVaR based on Cornish-Fisher expansion.}

The core of traditional VaR research is to analyze the statistical distribution of return on financial assets, and use historical information to predict future earnings distribution. However, yield usually does not follow the assumption of distribution.

The tail of the real market returns generally shows the characteristic of "thick tail" which is larger than the distribution. The normal distribution can be corrected by the Cornish Fisher method. It can be suitable for the actual situation of the financial fund field. Through data mining, we can find out the total income of the specific fund in the past 5 years. VaR Cornish-Fisher is used in the model, and the result is assumed to be normal distribution $V a R$. Compare the value and demonstrate the COrnish-Fisher extension method from an empirical perspective $V a R$. In the estimation, the Cornish-Fisher extension method based on data characteristics is effectively improved $V a R$ valuation.

The basic idea of the Cornish-Fisher method is that any empirical distribution can be represented by normal distribution. It is proposed by Cornish and Fisher. In the calculation of VaR, Cornish-Fisher extension method is used to correct VaR under normal assumptions.

The Cornish-Fisher expansion will combine the proceeds after standardization. $r^{*}$ percentile $\alpha$ approximate representation $q$ for:

$$
q=c(a)+\frac{1}{6}\left[c(a)^{2}-1\right]+\frac{1}{24}\left[c(a)^{3}-3 c(a)\right]\left(k_{p}-3\right)-\frac{1}{36}\left[2 c(a)^{3}-5 c(a)\right] s_{p}^{2}
$$

Among them:

$\mu_{p}$ means of portfolio returns, $\sigma_{p}$ is standard deviation of portfolio returns, $c(a)$ normal distribution $\alpha$ percentile $s_{p}$ bias for portfolio returns. $k_{p}$ the kurtosis of portfolio returns.

For normal distribution, its skewness is 0 and kurtosis is 3. $q=c(a)$ is non normal distribution, skewness and

kurtosis deviate from 0 and 3 respectively. $q$ not equal to $c(a)$

Therefore, portfolio benefit $r$ percentile $\alpha$ approximate: $\mu_{p}+\sigma_{p} q$ corresponding confidence level $1-\alpha$ of $\operatorname{VaR}$ for:

$$
\operatorname{VaR}(1-\alpha)=-\left(\mu_{p}+\sigma_{p} q\right)
$$

confidence level $1-\alpha$ of $C V a R$ for:

$$
C \operatorname{VaR}(1-\alpha)=-\sigma_{p}\left[M_{1}+\frac{1}{6}\left(M_{2}-1\right) s_{p}+\frac{1}{24}\left(M_{3}-3 M_{1}\right)\left(k_{p}-3\right)-\frac{1}{36}\left(2 M_{3}-5 M_{1}\right) s_{p}{ }^{2}\right]-\mu_{p}
$$

Among them:

$$
M_{i}=\frac{1}{\alpha} \int_{-\infty}^{c(a)} x^{i} f(x) d x, i=1,2,3
$$

Among them: $f(\square)$ is a probability density function of standard normal distribution.

Next, we still call Chang Bao's annual yield of ten thousand days in the past 5 years, and calculate the value of VaR and CVaR under Cornish-Fisher expansion.

This paper still uses the data of the balance treasure in recent five years, and calculates the value of VaR and CVaR through the CF expansion. From the diagram, we can see that the general trend is still similar, and there are some small fluctuations between the crest and the trough. This is also one of the places where CF expansion is superior to the historical simulation method, which is more sensitive to data and better depicts the small range changes.

\section{5. extreme value theory and VaR and CVaR}

\subsection{1. extreme value theory}

There are very short risks in all fields of financial investment, credit and insurance, that is, extreme losses will occur when extreme disasters occur. Therefore, for investors and risk managers, extreme risk loss estimation and probability prediction is a very important issue.

The traditional VaR computing method needs the distribution function of known return rate, which often leads to tail estimation. In theory, it is usually assumed that the distribution of return satisfies the elliptic distribution of normal, logarithmic or $t$ distribution. However, the empirical analysis shows that the distribution of financial asset returns is asymmetric, biased and higher kurtosis.Generally, the middle distribution is better, but the tail fitting is not good, so it may not be able to accurately predict the major risks that have never occurred in historical data. This requires a precise estimation of the tail distribution. The extreme 
value theory originated in the late 1990s provides a powerful tool for this. It is a good method for modeling the tail.

On the whole, the extreme theory model can be divided into 2 categories: one is the relatively old model, the interval maximum value model, which is based on the maximum and minimum observation values in a large number of independent sampling intervals (block); the other new extreme value theory model is higher than the threshold vertex model (POT). In this model, a threshold should be defined. It is a widely applied extreme value theory model to build models on all observation values above this threshold. The text uses the POT model in extreme value theory to calculate.

Considering the random variable $\mathrm{x}$, its probability density function and cumulative distribution function are respectively $f(x)$ and $F(x), X_{1}, X_{2}, \cdots, X_{n}$ a sequence of independent and identically distributed random variables.

Definition:

$$
\begin{aligned}
& X_{\text {max }, n}=\max \left(X_{1}, X_{2}, \cdots, X_{n}\right) \\
& X_{\min , n}=\min \left(X_{1}, X_{2}, \cdots, X_{n}\right)
\end{aligned}
$$

$$
H_{\max }\left(X_{\max , n}, \xi_{\max , n}, \mu_{\max , n}, \sigma_{\max , n}\right)=\exp \left\{-\left[1+\xi_{\max , n}\left(\frac{X_{\max , n}-\mu_{\max , n}}{\sigma_{\max , n}}\right)\right]^{-1 / \xi_{\max , n}}\right\}
$$

Among them:

$$
1+\xi_{\max , n}\left(\frac{X_{\max , n}-\mu_{\max , n}}{\sigma_{\max , n}}\right) \geq 0
$$

The corresponding probability density function is:

$$
\begin{gathered}
h_{\max }\left(X_{\max , n}, \xi_{\max , n}, \mu_{\max , n}, \sigma_{\max , n}\right)= \\
\frac{1}{\sigma_{\max , n}}\left[1+\xi_{\max , n}\left(\frac{X_{\max , n}-\mu_{\max , n}}{\sigma_{\max , n}}\right)\right]^{\frac{-1}{\xi_{\max , n}}-1} \exp \left\{-\left[1+\xi_{\max , n}\left(\frac{X_{\max , n}-\mu_{\max , n}}{\sigma_{\max , n}}\right)\right]^{-1 / \xi_{\max , n}}\right\}
\end{gathered}
$$

Among them: $X_{\max , n}$ asymptotic distribution can also be generalized Pareto distribution (GPD). $G_{\max }(x)$ Namely

$$
G_{\max }(x)=1+\ln \left(H_{\max }\right)
$$

to represent (Pickands, 1975)

$$
G_{\max }\left(X_{\max , n}, \xi_{\max , n}, \mu_{\max , n}, \sigma_{\max , n}\right)=1-\left[1+\xi_{\max , n}\left(\frac{X_{\max , n}-\mu_{\max , n}}{\sigma_{\max , n}}\right)\right]^{-1 / \xi_{\max , n}}
$$

Correspondingly, its probability density function is:

$$
g_{\max }\left(X_{\max , n}, \xi_{\max , n}, \mu_{\max , n}, \sigma_{\max , n}\right)=\frac{1}{\sigma_{\max , n}}\left[1+\xi_{\max , n}\left(\frac{X_{\max , n}-\mu_{\max , n}}{\sigma_{\max , n}}\right)\right]^{\frac{-1}{\xi_{\max , n}-1}}
$$




\subsubsection{Parameter estimation method of asymptotic distribution}

(1) nonlinear regression method

Gumbel (1958) proposed the use of nonlinear regression to estimate the parameters of the generalized extreme value distribution and the generalized Pareto distribution.

Known $_{X_{\max , n}} \mathrm{~N}$ observations: $X_{\max }^{1}, X_{\max }^{2}, \cdots, X_{\max }^{N}$ The order statistics sequence is $X_{\max }^{1}, X_{\max }^{2}, \cdots X_{\max }^{N}$ And satisfy

$$
X_{\max }^{1} \leq X_{\max }^{2} \leq \cdots \leq X_{\max }^{N} \quad \text { Obviously, }
$$

$X_{\max }^{r}, r=1,2, \cdots, N$ is a random variable, its probability density function is:

$$
f(x ; N, r)=\frac{x^{r-1}(1-x)^{N-r}}{B(r, N-r+1)}, 0 \leq x \leq 1
$$

Among them: $B(\square)$ isbeta function.

Correspondingly, $X_{\max }^{r}$ The mean value is

$$
\int_{0}^{1} \frac{x^{r}(1-x)^{N-r}}{B(r, N-r+1)} d x=\frac{r}{N+1}
$$

Alike, $H_{\max }\left(X_{\max }^{r}\right)$ is also a random variable on the $[0,1]$ interval, and its mean value is:

$$
E\left[H_{\max }\left(X_{\max }^{r}\right)\right]=E\left[\exp \left(-\left[1+\xi_{\max , n}\left(X_{\max }^{r}-\mu_{\max , n}\right) / \sigma_{\max , n}\right]^{-1 / \xi_{\max , n}}\right)\right]
$$

order

$$
E\left[\exp \left(-\left[1+\xi_{\max , n}\left(X_{\max }^{r}-\mu_{\max , n}\right) / \sigma_{\max , n}\right]^{-1 / \xi_{\max , n}}\right)\right]=\frac{r}{N+1}, r=1,2, \cdots, N
$$

We can establish the following regression equations to estimate the parameters of the generalized extreme value distribution.

$$
y=-\frac{1}{\xi_{\max , n}} \ln \left[1+\frac{\xi_{\max , n}\left(X_{\max }^{r}-\mu_{\max , n}\right)}{\sigma_{\max , n}}\right]+\varepsilon
$$

Among them: $y=-\ln \left(\frac{r}{N+1}\right)$

$$
E\left[G_{\max }\left(X_{\max }^{r}\right)\right]=E\left[1-\left[1+\xi_{\max , n}\left(X_{\max }^{r}-\mu_{\max , n}\right) / \sigma_{\max , n}\right]^{-1 / \xi_{\max , n}}\right]=\frac{r}{N+1}, \quad r=1,2, \cdots, N
$$

We can establish the following regression equations to estimate the parameters in the generalized Pareto distribution: $\left(\xi_{\max , n}, \mu_{\max , n}, \sigma_{\max , n}\right)$

$$
\begin{aligned}
& y=-\frac{1}{\xi_{\max , n}} \ln \left[1+\frac{\xi_{\max , n}\left(X_{\max }^{r}-\mu_{\max , n}\right)}{\sigma_{\max , n}}\right]+\varepsilon \\
& \text { Among them: } y=-\ln \left(1-\frac{r}{N+1}\right)
\end{aligned}
$$

Given observations $\left(\ln \left(1-\frac{r}{N+1}\right), X_{\max }^{r}\right), r=1,2, \cdots, N$,

$$
\ln L_{G}=-N \ln \sigma_{\max , n}-N\left(\frac{1+\xi_{\max , n}}{\xi_{\max , n}}\right) \sum_{i=1}^{N} \ln \left[1+\frac{\xi_{\max , n}\left(X_{\max }^{r}-\mu_{\max , n}\right)}{\sigma_{\max , n}}\right]
$$

The upper likelihood function is maximized to obtain the parameter estimation of the generalized Pareto distribution. 


\subsubsection{Calculation of VaR and CVaR by Extreme Value Theory}

$\mathrm{N}$ observation sequences with a given portfolio yield: $r_{1}, r_{2}, \cdots r_{N}$ We define the yield above the critical value $\mathrm{K}$ as the extreme value. $X_{\text {max }, n}$ For example, we can define the yield of 2 standard deviations over the mean. $X_{\max , n}$ We have a series of yields above K. $y_{1}, y_{2}, \cdots, y_{n}$ That is to say, there are more than $\mathrm{n}$ critical values $\mathrm{K}$. in $\mathrm{N}$ observations.

Further, we define new sequences. $z_{1}, z_{2}, \cdots, z_{n}$ For extremal sequences, here $z_{1}=y_{1}-K$, $z_{2}=y_{2}-K, \cdots, z_{n}=y_{n}-K$ By applying this sequence, we can estimate the parameters of generalized extreme value distribution or generalized Pareto distribution. After estimating these parameters, we can calculate the combined $\mathrm{VaR}$ and $\mathrm{CVaR}$. principles as follows:

Definition:

$$
\begin{gathered}
P\left(r_{t} \leq K\right)=F(K) \\
P\left(r_{t} \leq K+\mathrm{z}_{\mathrm{t}}\right)=F\left(K+\mathrm{z}_{\mathrm{t}}\right) \\
F_{\text {max }}\left(z_{t}\right)=\frac{F\left(K+z_{t}\right)-F(K)}{1-F(K)}
\end{gathered}
$$

When the critical value $\mathrm{k}$ is higher, $F_{\max }\left(z_{t}\right)$ It should be close to the generalized extreme value distribution or the generalized Pareto distribution.

First consider $F_{\text {max }}\left(z_{t}\right)$ It is close enough to the case of generalized extreme value distribution.

$$
\begin{aligned}
& \text { use } n / N \text { Act as } 1-F(K) \text { The estimate, we get: } \\
& F_{\max }\left(z_{t}\right)=\exp \left\{-\left[1+\frac{\xi\left(z_{t}-\mu\right)}{\sigma}\right]^{-\frac{1}{\xi}}\right\}=\frac{F\left(K+z_{t}\right)-(1-n / N)}{n / N}
\end{aligned}
$$

So:

$$
1-F\left(K+z_{t}\right)=\frac{n}{N}\left[1-\exp \left\{-\left[1+\frac{\xi\left(z_{t}-\mu\right)}{\sigma}\right]^{-\frac{1}{\xi}}\right\}\right]
$$

order

$$
\frac{n}{N}\left[1-\exp \left\{-\left[1+\frac{\xi\left(z_{t}-\mu\right)}{\sigma}\right]^{-\frac{1}{\xi}}\right\}\right]=1-\alpha
$$

obtain

$$
\begin{gathered}
\operatorname{VaR}_{z}^{G E V}(a)=\mu+\frac{\sigma}{\xi}\left\{\left[-\ln \left(1-(1-\alpha)^{N} / n\right)\right]^{-\xi}-1\right\} \\
\operatorname{VaR}_{r}(\alpha)=\operatorname{VaR}_{z}^{G E V}(a)+K
\end{gathered}
$$

Among them: $\operatorname{VaR}_{r}(\alpha)$ representing the distribution of portfolio returns $\alpha$ Confidence level $\operatorname{VaR}, \operatorname{VaR}_{z}^{G E V}(a)$ representing extreme value distribution $\alpha$ confidence level $\operatorname{VaR}$.

Correspondingly, we get $\mathrm{CVaR}_{r}(a)$ for:

$$
\operatorname{CVaR}_{r}(a)=K+\frac{1}{1-\alpha} \int_{0}^{1-\alpha} \operatorname{VaR}_{z}^{G E V} x d x
$$

Next we will consider $F_{\max }\left(z_{t}\right)$ is close enough to the case of generalized Pareto distribution. Similar results can be obtained.

$$
\begin{gathered}
\operatorname{VaR}_{z}^{G P D}(\alpha)=\mu+\frac{\sigma}{\xi}\left\{\left[(1-\alpha)^{N} / n^{-\xi}-1\right\}\right. \\
\operatorname{VaR}_{r}(\alpha)=\operatorname{VaR}_{z}^{G P D}(\alpha)+K \\
\operatorname{CVaR}_{r}(\alpha)=K+\frac{1}{1-\alpha} \int_{0}^{1-\alpha} \operatorname{VaR}_{z}^{G P D} x d x
\end{gathered}
$$

Notice what we get from above. $\operatorname{VaR}_{r}(\alpha)$ and $\operatorname{CVaR}_{r}(\alpha)$ is based on the right tail. $\operatorname{VaR}_{r}(\alpha)$ and $\operatorname{CVaR}_{r}(\alpha)$ if you want to get the left tail. $\operatorname{VaR}_{r}(\alpha)$ and $\operatorname{CVaR}_{r}(\alpha)$ We only need to observe the yield of $\mathrm{N}$ observation sequence: $r_{1}, r_{2}, \cdots, r_{n}$ Change to $-r_{1},-r_{2}, \cdots,-r_{n}$ the rest of the steps remain unchanged based on the left tail $\operatorname{VaR}_{r}(\alpha)$ and $\operatorname{CVaR}_{r}(\alpha)$

Next, we use the above GPD based nonlinear regression method to estimate the combined returns VaR and CVaR to calculate the change of VaR and CVaR of Guizhou Moutai in the last 5 years in the A share market as at the highest single stock price in September 1, 2019.

As shown in the figure:

To better contrast, we use the historical simulation method to calculate the VaR and CVaR values with a confidence level of $95 \%$.

\section{Conclusion}

It can be seen that the overall trend is similar, but there are some differences in the specific degree of risk aversion. And from the two images, we can see that the overall risk of Moutai is relatively low. In a longer period of time, the probability of Moutai losing more than three percent is less than five percent. From this single indicator, we can see that Moutai is a stock worth holding for a long time and suitable for value investment.

\section{References}

[1] Le, J.J. (2014) Research on the fluctuation characteristics and risk of gold futures market based on VaR-GARCH model D. Anhui University.

[2] Liang, H. (2014) Research on Asymmetric Volatility of Shanghai and Shenzhen stock market based on GARCH family model D. Anhui University.

[3] Cheng, C. (2014) Analysis of Shanghai stock index volatility characteristics based on GARCH model D. Shandong University.

[4] Pan, J. (2102) Calculation of Shanghai and Shenzhen index VaR and CVaR based on GARCH model D. Wuhan University of Science and Technology. 